\title{
Tuning the Composition of Alloy Nanoparticles through Laser Mixing: the Role of Surface Plasmon Resonance
}

Gabriele C. Messina, ${ }^{*}{ }^{\dagger}$ Marco G. Sinatra, ${ }^{\dagger}$ Valentina Bonanni, ${ }^{\S}, \|$ Rosaria Brescia, ${ }^{\perp}$ Alessandro Alabastri, ${ }^{\#}$ Francesco Pineider, ${ }^{\S, \Upsilon, \bullet}$ Giulio Campo, ${ }^{\S}$ Claudio Sangregorio," Giovanni Li-Destri, ${ }^{\Delta}$ Gianfranco Sfuncia, ${ }^{\nabla}$ Giovanni Marletta, ${ }^{\mathbf{2}}$ Marcello Condorelli, ${ }^{\dagger}$ Remo Proietti Zaccaria, ${ }^{\dagger}$ Francesco De Angelis, ${ }^{\dagger}$ and Giuseppe Compagnini ${ }^{\dagger}$

${ }^{\dagger}$ Plasmon Nanotechnologies and ${ }^{\perp}$ Nanochemistry Department, Istituto Italiano di Tecnologia, Via Morego 30, 16163 Genoa, Italy

${ }^{\dagger}$ Laboratory for Thin Films and Nanostructures (LABFSN), Department of Chemical Sciences, University of Catania, Viale Andrea Doria 6, 95124 Catania, Italy

${ }^{\S}$ Department of Chemistry, University of Florence \& INSTM, Via della Lastruccia 3, 50019 Sesto Fiorentino (FI), Italy

"Department of Physics, University of Milan \& CNR-IOM, Trieste, I-34149, Italy

\#Department of Physics and Astronomy MS 61, Laboratory for Nanophotonics, Smalley-Curl Institute, Rice University, Houston, Texas 77005, United States

${ }^{\mathbb{I}}$ Department of Chemistry and Industrial Chemistry, University of Pisa, Via Moruzzi 3, 56124 Pisa, Italy

"ICCOM-CNR, Sesto Fiorentino, Florence, I-50019, Italy $\triangle$ European Synchrotron Radiation Facility, 71 Avenue des Martyrs, 38000 Grenoble, France

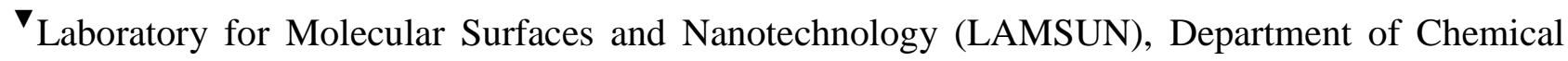
Sciences, University of Catania and CSGI, Viale Andrea Doria 6, 95124, Catania, Italy

*E-mail: gabriele.messina@iit.it 


\section{Structural Characterization}

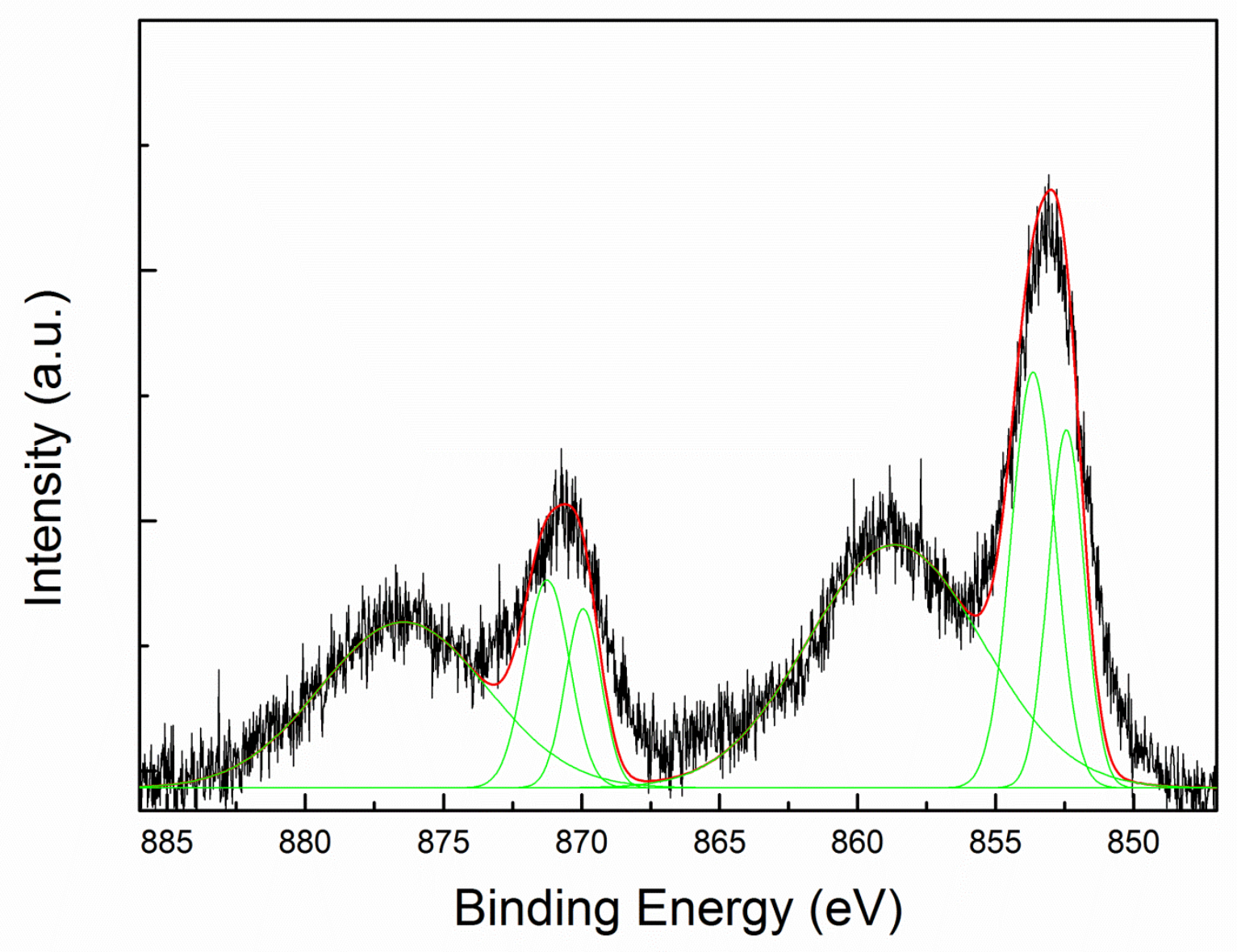

Figure S1. XPS spectrum of Ni2p of as prepared Ni@NiO colloidal solution.

Figure $\mathrm{S} 1$ reports the Ni2p spectrum of a solution obtained from the ablation of a Ni target in water. It is possible to notice the presence of the $\mathrm{NiO}$ component $2 \mathrm{p}^{3 / 2}$ at higher binding energy $(853.6 \mathrm{eV})$ with respect to metallic $\mathrm{Ni} 2 \mathrm{p}^{3 / 2}(852.5 \mathrm{eV})$. Moreover, the component at $856.2 \mathrm{eV}$ is attributed to $\mathrm{Ni}(\mathrm{OH})_{2 .}{ }^{\ddagger}$. The other signals in the spectrum are related to satellite peaks of $\mathrm{NiO}, \mathrm{Ni}(\mathrm{OH})_{2}$. Integration of the peak areas reports that the oxide component represents the $60 \%$ of the whole amount of Ni.

${ }^{\ddagger}$ Nesbitt, H. W.; Legrand, D.; Bancroft, G. M. Phys Chem Minerals 2000, 27, 357 


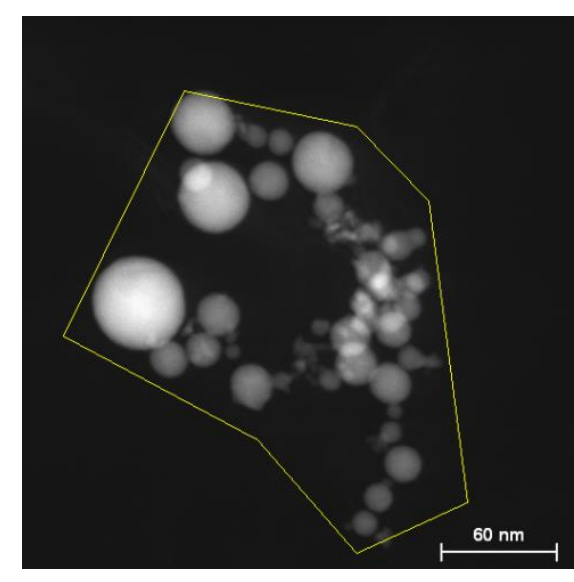

$\mathrm{Au} / \mathrm{Ni} / \mathrm{O}=58 / 14 / 28$
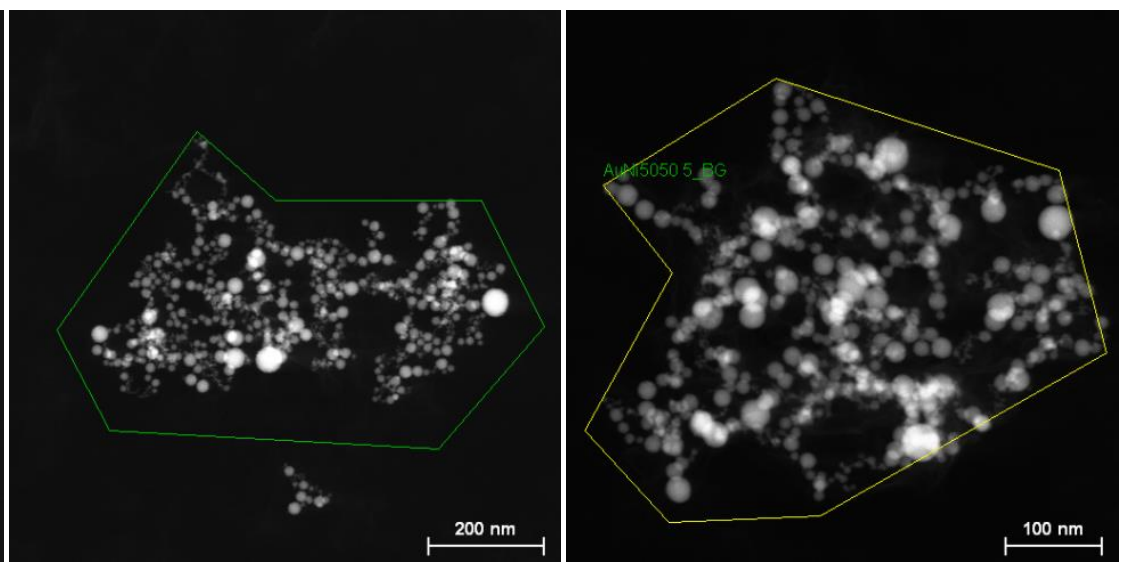

$\mathrm{Au} / \mathrm{Ni} / \mathrm{O}=88 / 12 / 0$

$\mathrm{Au} / \mathrm{Ni} / \mathrm{O}=48 / 31 / 21$

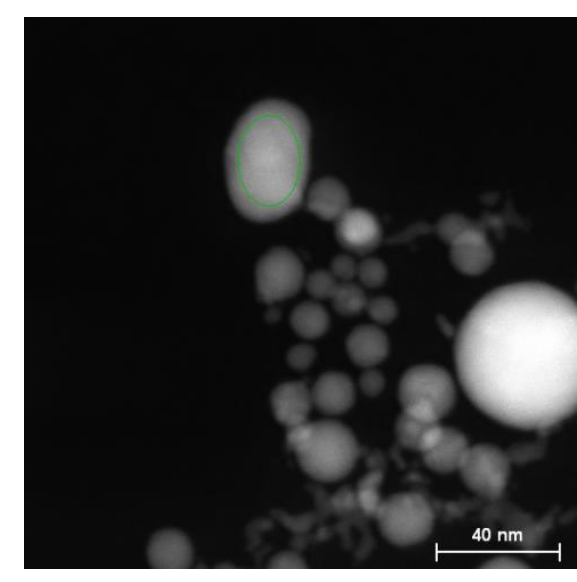

$\mathrm{Au} / \mathrm{Ni} / \mathrm{O}=93 / 7 / 0$

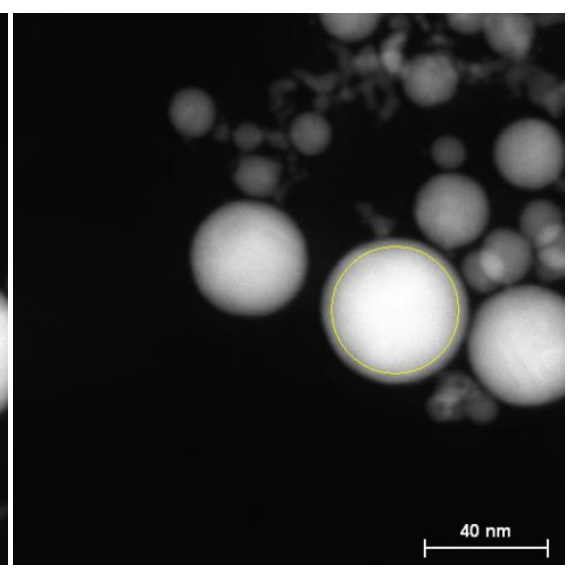

$\mathrm{Au} / \mathrm{Ni} / \mathrm{O}=92 / 8 / 0$

Figure S2. EDS quantification (atomic \%) of the average elemental composition performed on $\mathrm{Au} / \mathrm{Ni}$ 50/50 particles.

Figure S2 shows EDS elemental analysis on the colloidal solution obtained for Au/Ni 50/50 starting mixture. It is possible to observe that analysis performed on large areas of the samples showed the presence of oxygen. Analysis performed on single large particles showed only the presence of gold and nickel, while in smaller particle also oxygen was found. On the base of this results and average background with a composition of $\mathrm{Au} / \mathrm{Ni} / \mathrm{O}=65 / 19 / 16$ has been subtracted leading to the statistic results reported in the paper. 

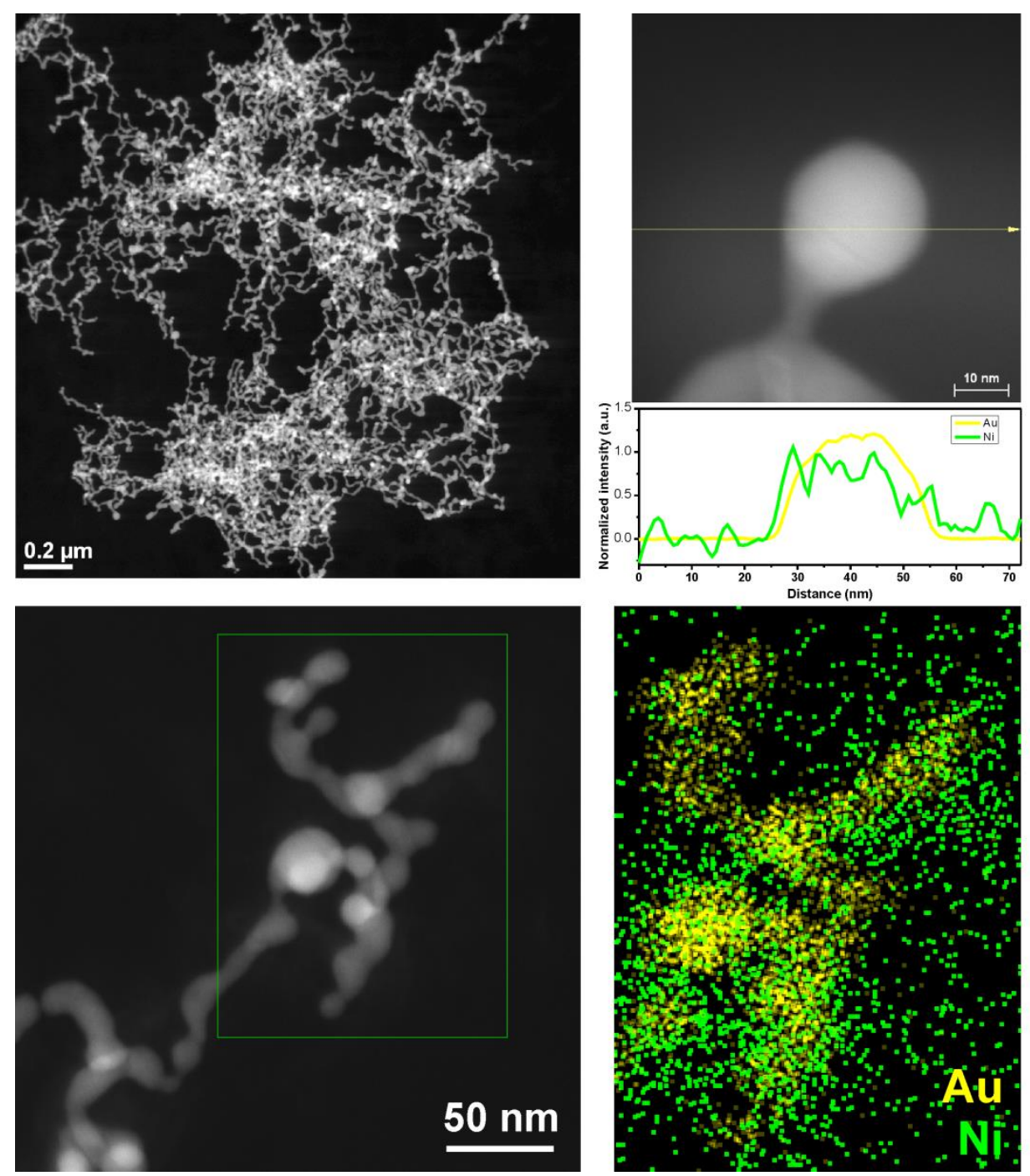

Figure S3. HAADF-STEM imaging and EDS analysis performed on Au/Ni 67/33 particles. 

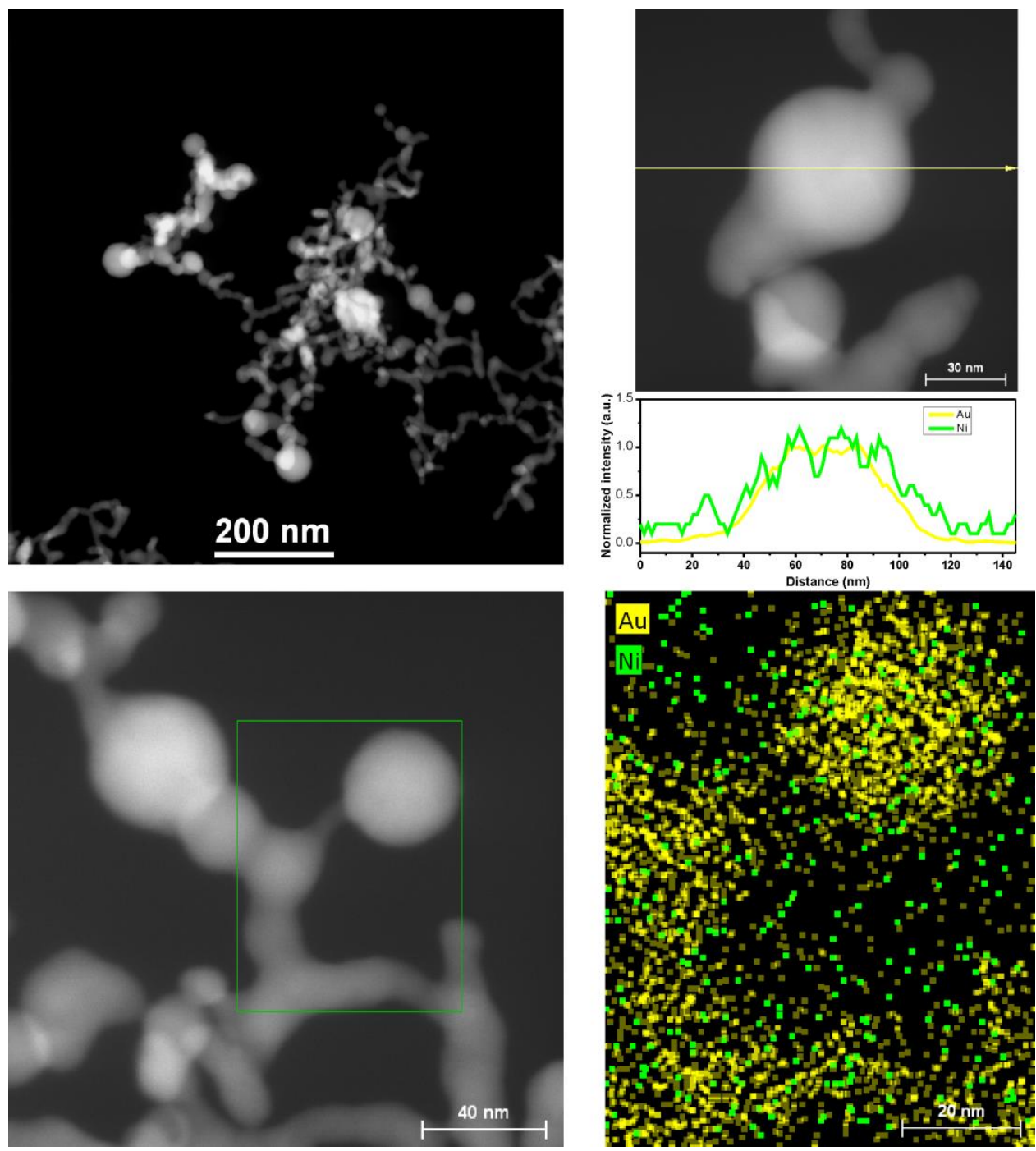

Figure S4. HAADF-STEM imaging and EDS analysis performed on Au/Ni 75/25 particles. 

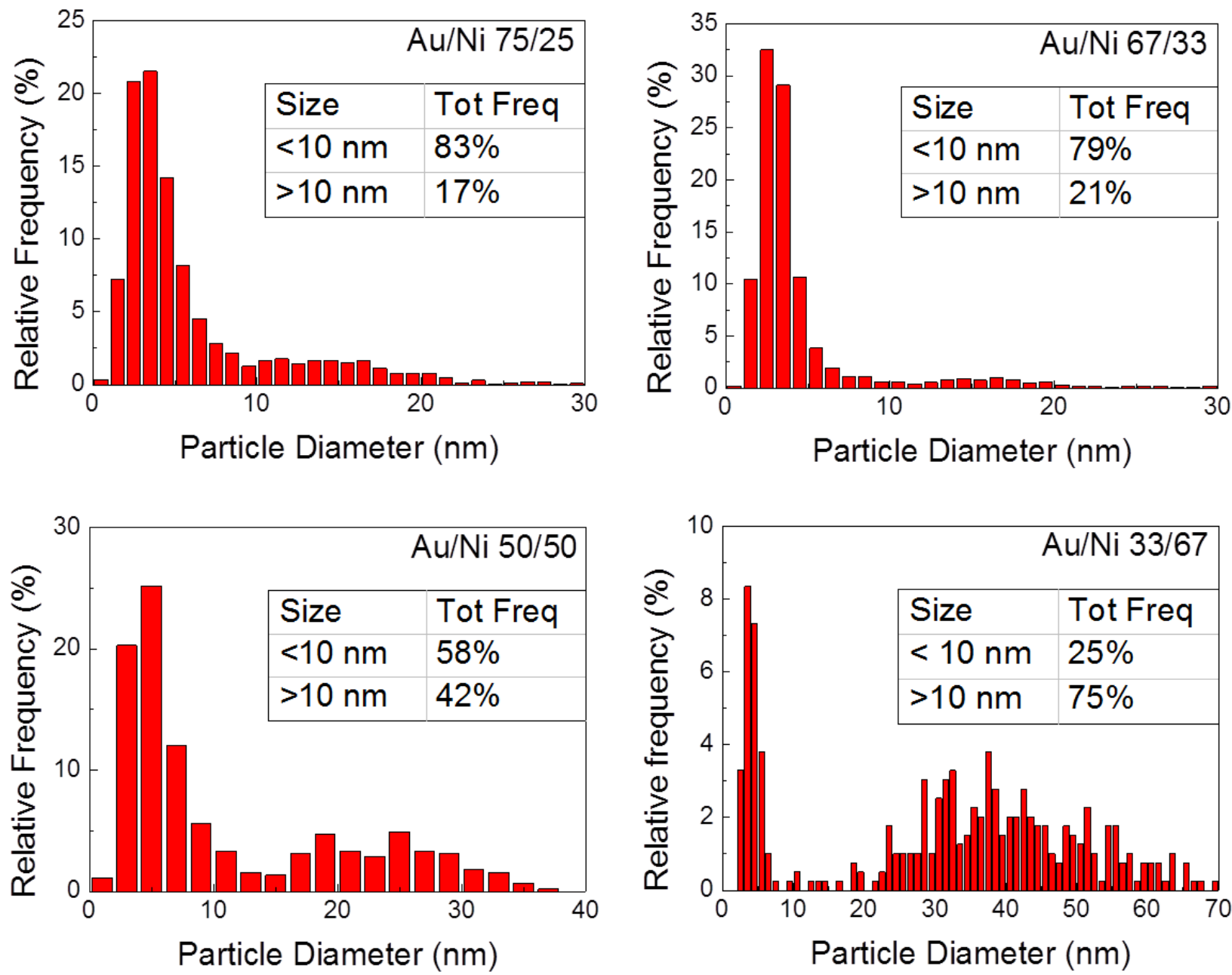

Figure S5. Particle size distributions of the obtained samples. Tot Freq (Total Frequency) refers to the number of particles with determined size with respect to the whole number of particles in the sample. 


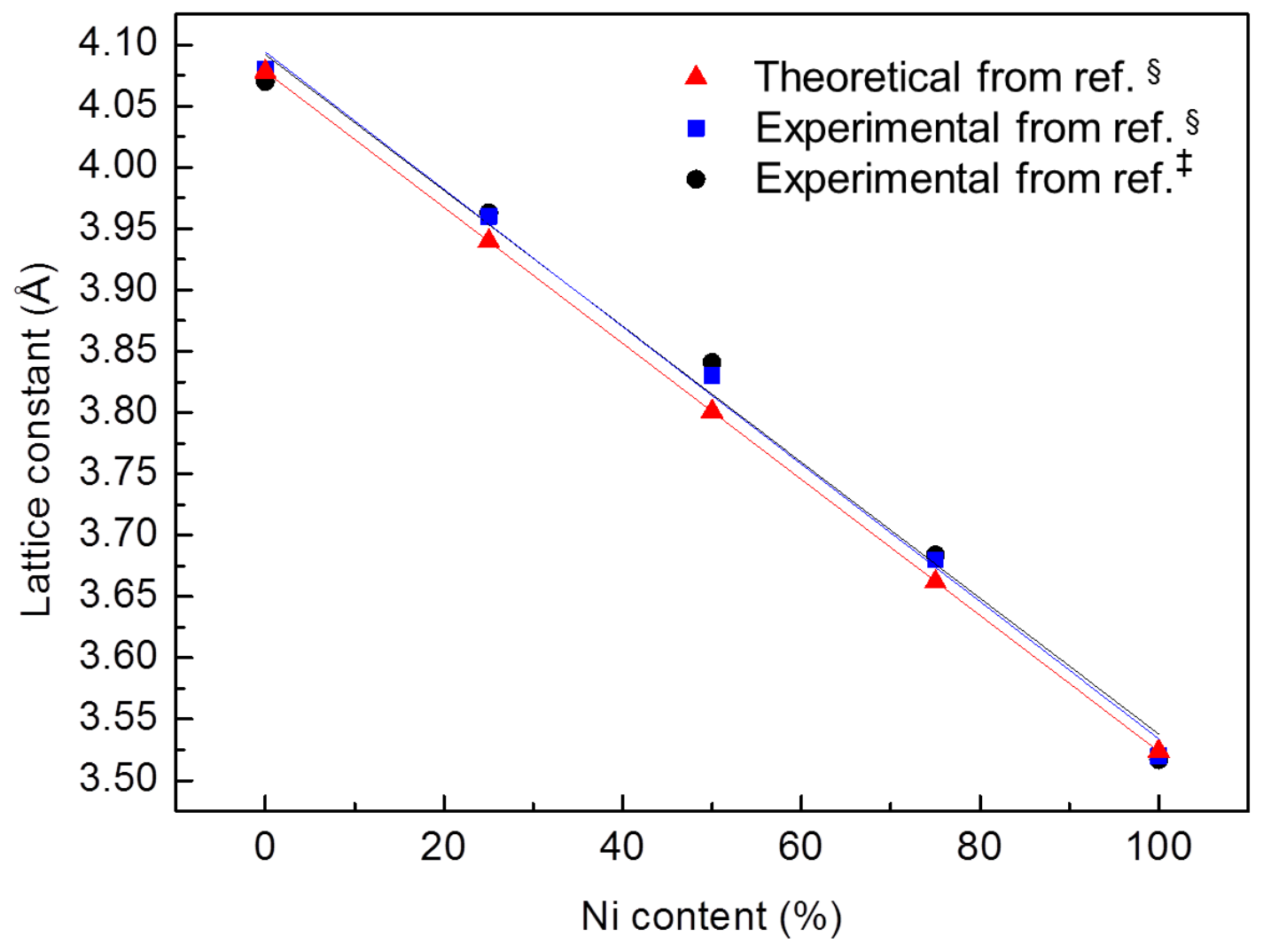

Figure S6. Lattice constant of a $\mathrm{Au}_{\mathrm{x}} \mathrm{Ni}_{1-\mathrm{x}}$ alloy as a function of the nickel content.

Data from literature refer to:

$\S$ Tsaur, B.Y.; Mäenpää, M. J. Appl. Phys., 1981, 52, 728-735

${ }^{\ddagger}$ Ellwood, E.C.; Bagley, K.Q.; J. Inst. Metals, 1951-1952, 80, 617 


\section{Optical and Magnetic Characterization}

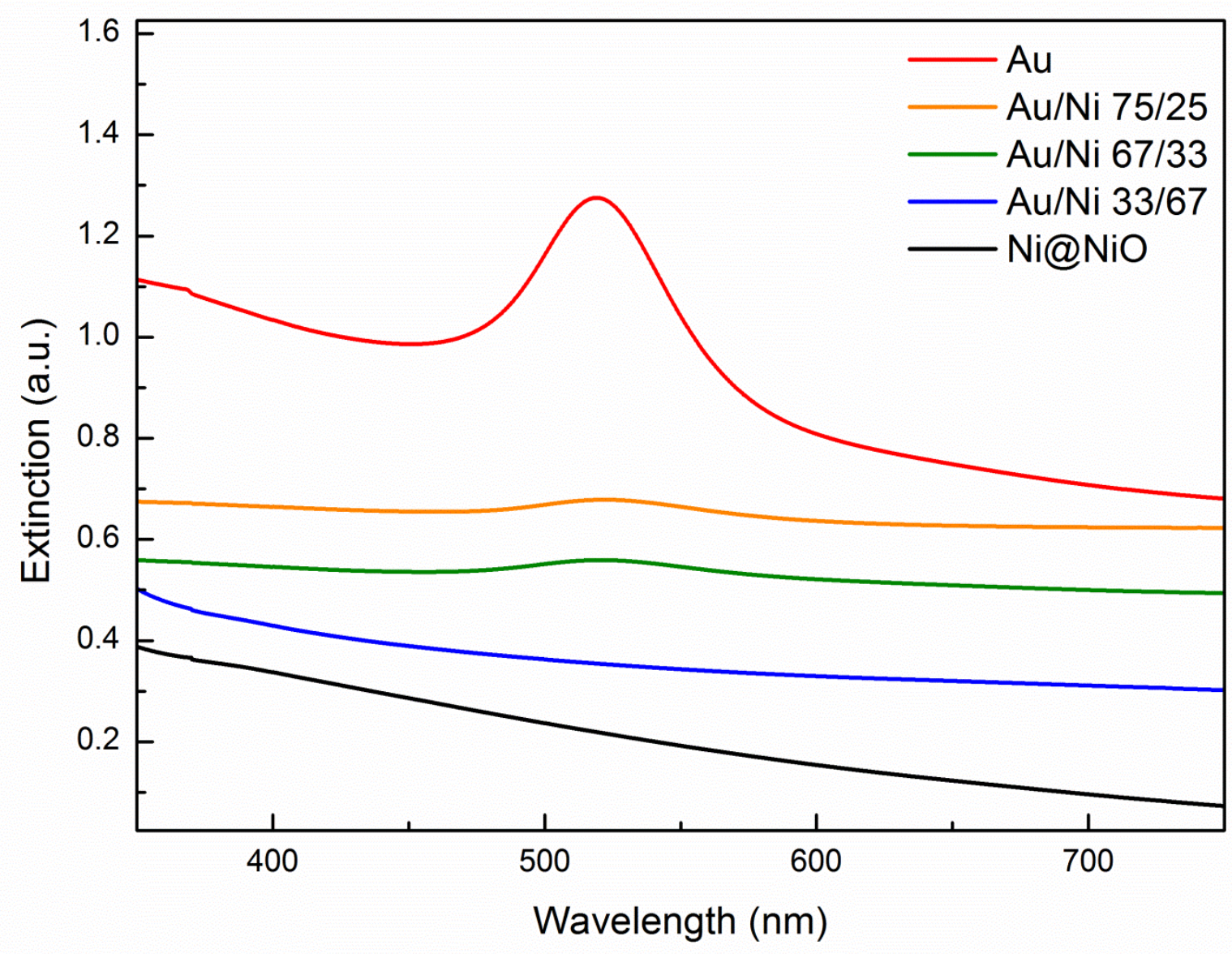

Figure S7. UV-Vis spectra of colloidal mixtures with different composition.
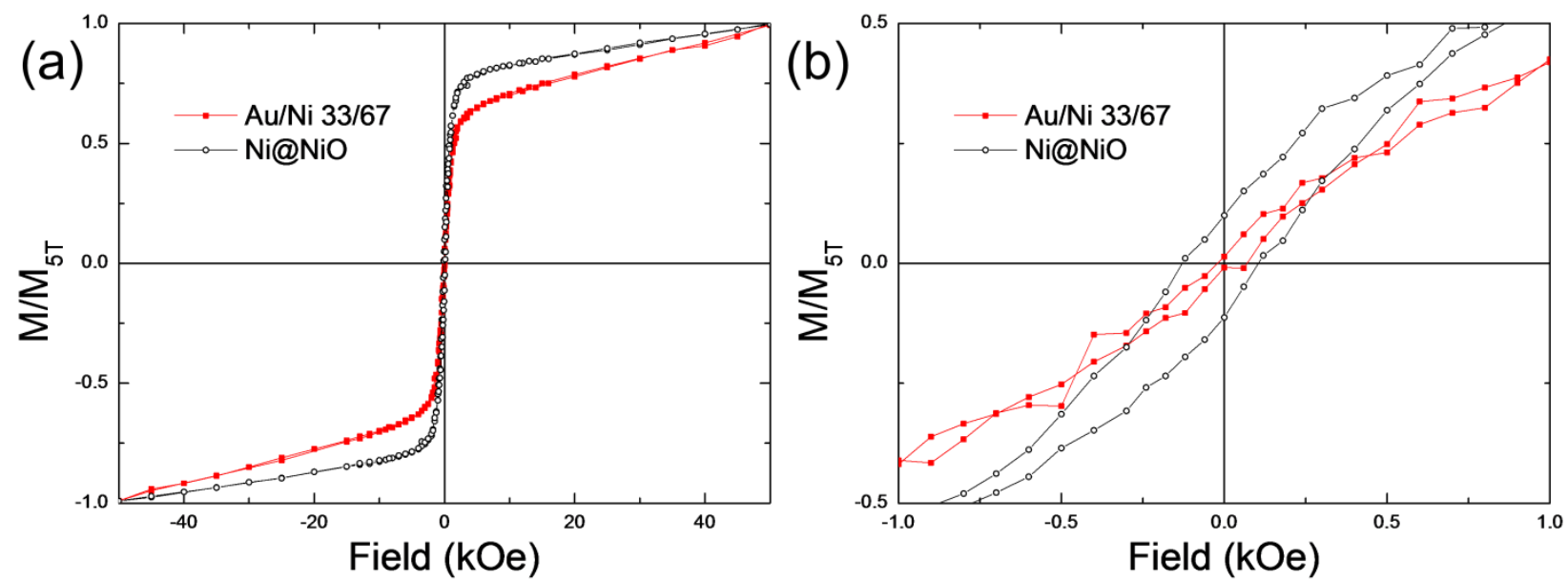

Figure S8. Hysteresis loop at $300 \mathrm{~K}$ obtained for (a) Ni@ NiO and Au/Ni 33/67 and (b) zoom of the hysteresis curves shown in (a). 